ANNALES

POLONICI MATHEMATICI

$84.2(2004)$

\title{
$\nabla$-flat functions on manifolds
}

\author{
by WoJCIECH KozŁOWski (Łódź)
}

\begin{abstract}
We investigate $\nabla$-flat and pointwise- $\nabla$-flat functions on affine and Riemannian manifolds. We show that the set of all $\nabla$-flat functions on $(M, \nabla)$ is a ring which has interesting properties similar to the ring of polynomial functions.
\end{abstract}

1. Introduction, preliminaries and primary results. This paper is devoted to some aspects of the connection theory, namely we consider an affine manifold $(M, \nabla)$ and investigate the set of $\nabla$-flat and pointwise- $\nabla$-flat functions. Although $\nabla$ is an elliptic operator (see [We] or [Na]), we use only elementary methods of differential geometry.

In this section we prepare our tools. The major one is Proposition 1.1 which seems to be of independent interest. Moreover, it is shown that if $(M, \nabla)$ is real analytic then each flat function on $M$ is also real analytic (see Corollary 1.1).

The main results are given in Section 2. We prove there (see Theorem 2.1) that if $(M, \nabla)$ is real analytic and $M$ is connected then any pointwise- $\nabla$-flat function is $\nabla$-flat and real analytic. We also show that the ring of $\nabla$-flat functions on any smooth and connected manifold is an integral domain. Moreover (see Theorem 2.2), it is proved that each $\nabla$-flat and bounded function on any complete Riemannian manifold is constant. In particular, if $M$ is connected and compact then each $\nabla$-flat function must be constant.

Let $(M, \nabla)$ be an affine manifold. For any integer $k \geq 0$ put $\nabla^{k}=$ $\nabla \circ \cdots \circ \nabla$ ( $k$ times). A function $f \in C^{\infty}(M)$ is called $\nabla$-flat if $\nabla^{k} f=0$ for some $k \geq 0$. A function $f \in C^{\infty}(M)$ is called pointwise- $\nabla$-flat if for each $x \in M$ there exists $k=k(x) \geq 0$ such that $\left(\nabla^{k} f\right)(x)=0$. A smooth function $f$ on a Riemannian manifold $(M, g)$ is called $\nabla$-flat if $f$ is $\nabla$-flat with respect to Levi-Civita's connection $\nabla$ on $M$. Moreover, we will say that $f$ is $k-\nabla$-flat if $\nabla^{k} f=0$. Obviously, for any $k$ the set $\mathcal{P}_{k}(M, \nabla)$ of all

2000 Mathematics Subject Classification: 53C05, 53A15.

Key words and phrases: affine manifold, Riemannian manifold, $\nabla$-flat function, polynomial function. 
$k$ - $\nabla$-flat functions on $M$ is a vector space, whereas the set $\mathcal{P}(M, \nabla)$ of all $\nabla$-flat functions on $M$ is a ring.

ExAMPLE. Let $M$ be an open subset of $\mathbb{R}^{n}$ and $\nabla$ denote the canonical connection on $M$, i.e. $\nabla_{\partial / \partial x^{i}} \partial / \partial x^{j}=0$. Then one can easily verify that $f$ is $k$ - $\nabla$-flat if and only if $f$ is a polynomial function of degree $<k\left({ }^{1}\right)$.

Using the Baire property (cf. [He, Lemma 3.1]) one can prove the following

LEMmA 1.1. Consider a smooth function $f: I \rightarrow \mathbb{R}$, where $I \subset \mathbb{R}$ is an open interval. If for each $x \in I$ there exists an integer $k=k(x) \geq 0$ such that $f^{(k)}(x)=0$ then $f$ is a polynomial function on I. Here $f^{(k)}=\bar{d}^{k} f / d t^{k}$.

Let $(M, \nabla)$ be an affine manifold. Take $p \in M$, and let $G$ be an open and star-shaped neighbourhood of zero in $T_{p} M$ such that the exponential mapping exp at $p$ is defined on $G$. For any $v \in G$ let $\varphi_{v}: I \rightarrow M$ denote the geodesic curve such that $\varphi_{v}(I) \subset \exp (G), \varphi_{v}(0)=p$ and $\dot{\varphi}_{v}(0)=v$.

Proposition 1.1. Let $k \geq 0$. Suppose that $f$ is a smooth function on $M$. If $\varphi_{v}^{\star} \nabla^{k} f=0$ for each $v \in G$, then $f \circ \exp$ is a polynomial function on $G$ of degree $<k$.

Proof. If $k=0$ then the assertion is obvious. Suppose that $k \geq 1$. Set $v \in G$. Put $\varphi=\varphi_{v}$ for simplicity. Take $t_{0} \in I$. Let $I^{\prime} \subset I$ be a neighbourhood of $t_{0}$ such that $\varphi \mid I^{\prime}$ is an embedding. Put $I^{\prime \prime}=\varphi\left(I^{\prime}\right)$. Suppose $E$ is a vector field defined on an open neighbourhood of $I^{\prime \prime}$ in $M$ such that $E \circ \varphi=\dot{\varphi}$. Define now the affine connection $\nabla^{\prime \prime}$ on $I^{\prime \prime}$ by putting $\nabla_{E}^{\prime \prime} E=0$. Let $\nabla^{\prime}$ be the canonical connection on $I^{\prime}$. Since $\varphi$ is a geodesic curve, $\varphi^{\star} \nabla^{\prime \prime k} f=$ $\varphi^{\star} \nabla^{k} f=0$ on $I^{\prime \prime}$.

By the definitions of $\nabla^{\prime}$ and $\nabla^{\prime \prime}$ it follows immediately that $\varphi:\left(I^{\prime}, \nabla^{\prime}\right) \rightarrow$ $\left(I^{\prime \prime}, \nabla^{\prime \prime}\right)$ is an affine diffeomorphism. Hence $\nabla^{\prime k}(f \circ \varphi)=\varphi^{\star} \nabla^{\prime \prime k} f=0$. Since $\nabla^{\prime}$ is the canonical connection on $I^{\prime}$ (see Example above), $f \circ \varphi$ is a polynomial function on $I^{\prime}$ of degree $<k$. Since $t_{0}$ is arbitrary, $f \circ \varphi$ must be a polynomial function on $I$.

Consider now an arbitrary $v \in G$ and put $P=f \circ \exp$. Using the chain rule, we obtain

$$
P(v)-P(0)=\sum_{0<|\alpha|<k} \frac{1}{\alpha !} \frac{\partial^{|\alpha|} P}{\partial x_{1}^{\alpha_{1}} \ldots \partial x_{n}^{\alpha_{n}}}(0) v_{1}^{\alpha_{1}} \ldots v_{n}^{\alpha_{n}} .
$$

Hence $P$ is a polynomial function of degree $<k$.

$\left({ }^{1}\right)$ Throughout this paper the degree of the zero polynomial function is $-\infty$. 
A straightforward consequence of Proposition 1.1 is the following

THEOREM 1.1. Suppose $f$ is a $k-\nabla$-flat function on $M$. Then for each point $p \in M, f \circ \exp i s$ a polynomial function on $G$ of degree $<k$.

Corollary 1.1. Suppose $(M, \nabla)$ is a real analytic and $f \in C^{\infty}(M)$. If $f$ is $\nabla$-flat then $f$ is real analytic.

2. Main results. Let $(M, \nabla)$ be an affine manifold. Recall (see $[\mathrm{KN}$, Theorem 8.7(2)]) that each $q \in M$ has a neighbourhood $W$ such that any $p \in W$ has a normal neighbourhood containing $W$.

TheOREM 2.1. Suppose $(M, \nabla)$ is real analytic and $M$ is connected. If a smooth function $f$ on $M$ is pointwise- $\nabla$-flat then $f$ is real analytic and $\nabla$-flat.

Proof. Define an open set $V \subset M$ as follows: $p \in V$ if there exists an open neighbourhood, say $W_{p}$, of $p$ and an integer $k \geq 0$ such that $\nabla^{k} f=0$ on $W_{p}$. The Baire property implies that $V$ is dense. Corollary 1.1 implies that $f$ is real analytic and flat on each connected component of $V$.

Suppose that $E=M \backslash V \neq \emptyset$. Let $q \in E$. Take $p$ such that $f$ is $k$ - $\nabla$-flat on some neighbourhood of $p$ and a normal neighbourhood $\Omega$ of $p$ containing $q$. We may suppose that $\Omega=\exp (G)$, where $G$ is as in the previous section. Since $\exp : G \rightarrow \Omega$ is a real analytic diffeomorphism it suffices to show that $f \circ \exp$ is a polynomial function on $G$. Take any $v \in G$ and let $\varphi: I^{\prime} \rightarrow \Omega$ be a geodesic curve such that $\varphi(0)=p$ and $\dot{\varphi}(0)=v$. Put $I^{\prime \prime}=\varphi\left(I^{\prime}\right)$. Let $\nabla^{\prime \prime}$ be an affine connection on $I^{\prime \prime}$ defined as in the proof of Proposition 1.1. If $\nabla^{\prime}$ is the canonical connection on $I^{\prime}$ then $\varphi:\left(I^{\prime}, \nabla^{\prime}\right) \rightarrow\left(I^{\prime \prime}, \nabla^{\prime \prime}\right)$ is an affine diffeomorphism, hence for any $r \geq 0, \nabla^{\prime r}(f \circ \varphi)=\varphi^{\star} \nabla^{\prime \prime r} f$. Since $\varphi$ is a geodesic curve, one can easily check that $\varphi^{\star} \nabla^{\prime \prime r} f=\varphi^{\star} \nabla^{r} f$. This implies that the restriction of $f$ to $I^{\prime \prime}$ is pointwise- $\nabla^{\prime \prime}$-flat on $I^{\prime \prime}$ and $\nabla^{\prime \prime k} f=0$ on some neighbourhood of $p$ in $I^{\prime \prime}$, therefore $f \circ \varphi$ is pointwise- $\nabla^{\prime}$-flat on $I^{\prime}$ and $\nabla^{\prime k}(f \circ \varphi)=0$ on some open interval contained in $I^{\prime}$. Since $\nabla^{\prime}$ is the canonical connection on $I^{\prime}$, Lemma 1.1 implies now that $\nabla^{\prime k}(f \circ \varphi)=0$ on $I^{\prime}$. So $\varphi^{\star} \nabla^{k} f=\varphi^{\star} \nabla^{\prime \prime k} f=\nabla^{\prime k}(f \circ \varphi)=0$. Since $v \in G$ was arbitrary, Proposition 1.1 implies that $f \circ \exp$ is a polynomial function on $G$.

Suppose now that both $M$ and $\nabla$ are just smooth. Let $\mathcal{P}=\mathcal{P}(M, \nabla)$ and $\mathcal{P}_{k}=\mathcal{P}_{k}(M, \nabla)$.

Proposition 2.1 (Identity Principle). Let $M$ be connected and $f \in \mathcal{P}$. If $f=0$ on some open subset of $M$ then $f=0$ on $M$.

Proof. Define an open set $W \subset M$ as follows: $q \in W$ if $f=0$ on some neighbourhood of $q$. Since $W$ is nonempty and $M$ is connected, it suffices to show that $W$ is closed. Let $p$ be an accumulation point of $W, \Omega$ a normal neighbourhood of $p$ and $G=\exp ^{-1}(\Omega)$. Theorem 1.1 implies that 
$P=f \circ \exp$ is a polynomial function on $G$ and $P=0$ on a nonempty open subset $\exp ^{-1}(W)$ of $G$. Hence $P=0$ on $G$, so $f=0$ on $\Omega$. Thus $p \in W$.

Corollary 2.1. If $M$ is connected then $\mathcal{P}$ is an integral domain.

COROLlary 2.2. If $M$ is connected then $\operatorname{dim} \mathcal{P}_{k}<d(n, k)$, where $d(n, k)$ denotes the dimension of the vector space $\mathcal{R}_{k}$ of all polynomial functions on $\mathbb{R}^{n}$ of degree $\leq k$.

Proof. Fix $p \in M$. Let $\Omega$ be a normal neighbourhood of $p$ and let $G=\exp ^{-1}(\Omega)$. Let $\mathcal{P}_{k}^{\Omega}$ denote the vector space of all restrictions of members of $\mathcal{P}_{k}$ to $\Omega$. Using Theorem 1.1 and identifying the space of all polynomial functions on $G$ of degrees $<k$ with $\mathcal{R}_{k}$ we see that $\exp ^{\star}: \mathcal{P}_{k}^{\Omega} \rightarrow \mathcal{R}_{k}$ is a vector space monomorphism. If $f_{1}, \ldots, f_{r}$ is a basis of $\mathcal{P}_{k}^{\Omega}$ then Proposition 2.1 implies that $f_{1}, \ldots, f_{r}$ is a basis of $\mathcal{P}_{k}$. Thus $\operatorname{dim} \mathcal{P}_{k}=r<d(n, k)$.

Let now $(M, g)$ be a connected Riemannian manifold and let $\nabla$ be LeviCivita's connection on $M$.

THEOREM 2.2 (Liouville type theorem). If $(M, g)$ is complete then each $\nabla$-flat and bounded function $f$ on $M$ is constant. In particular if $M$ is compact then each $\nabla$-flat function on $M$ is constant.

Proof. Since $(M, g)$ is a complete, the exponential mapping at $p$ is defined on the whole tangent space $T_{p}$. Since $f$ is bounded, Theorem 1.1 implies that $f$ must be constant on some neighbourhood of $p$. Theorem 2.2 is now a consequence of Proposition 2.1.

\section{References}

[He] S. Helgason, Differential Geometry, Lie Groups, and Symmetric Spaces, Amer. Math. Soc., 2001.

[KN] S. Kobayashi and K. Nomizu, Foundations of Differential Geometry, Wiley, New York, 1963.

[Na] R. Narasimhan, Analysis on Real and Complex Manifolds, North-Holland, Amsterdam, 1968.

[We] R. O. Wells, Differential Analysis on Complex Manifolds, Prentice-Hall, Englewood Cliffs, NJ, 1973.

Institute of Mathematics

Polish Academy of Sciences

Łódź Branch

Banacha 22

90-238 Łódź, Poland

E-mail: wojciech@math.uni.lodz.pl 